\title{
Single-center experience with the frozen elephant trunk procedure in 111 patients with complex aortic disease
}

\author{
Oliver J. Liakopoulos ${ }^{1,2}$, Axel Kroener ${ }^{1}$, Anton Sabashnikov, Mohamed Zeriouh ${ }^{1,2}$, Wael Ahmad ${ }^{3}$, \\ Yeong-Hoon Choi ${ }^{1,2}$, Thorsten Wahlers ${ }^{1}$ \\ ${ }^{1}$ Department of Cardiothoracic Surgery, Heart Center of the University Hospital of Cologne, Cologne, Germany; ${ }^{2}$ Department of Cardiac Surgery, \\ Kerckhoff Clinic, Bad Nauheim, Campus Kerckhoff, University of Giessen, Hessen, Germany; ${ }^{3}$ Department of Vascular Surgery, Heart Center of \\ the University Hospital of Cologne, Cologne, Germany \\ Contributions: (I) Conception and design: OJ Liakopoulos, YH Choi, T Wahlers; (II) Administrative support: OJ Liakopoulos, A Sabashnikov, W \\ Ahmad, M Zeriouh, A Kroener; (III) Provision of study materials or patients: OJ Liakopoulos, A Kroener, M Zeriouh, T Wahlers, YH Choi, T \\ Wahlers; (IV) Collection and assembly of data: OJ Liakopoulos, W Ahmad, A Sabashnikov; (V) Data analysis and interpretation: OJ Liakopoulos, W \\ Ahmad, A Sabashnikov, T Wahlers; (VI) Manuscript writing: All authors; (VII) Final approval of manuscript: All authors. \\ Correspondence to: Oliver J. Liakopoulos, MD. Professor, Department of Cardiothoracic Surgery, Heart Center of the University of Cologne, \\ Kerpener Strasse 62, 50924 Cologne, Germany. Email: oliver.liakopoulos@uk-koeln.de.
}

Background: The frozen elephant trunk (FET) technique is increasingly used for the treatment of acute and chronic aortic arch disease. This study reports our single center experience with the FET technique in patients with complex aortic disease.

Methods: Between 2009 and 2019, 111 consecutive patients underwent aortic arch surgery in our institution using the FET technique for acute type A dissection (AAD group; $n=75$ ) or non-acute type A dissection (non-AAD group; $\mathrm{n}=36 ; 10$ patients with chronic type A dissection; 26 patients with aneurysm), respectively. Relevant perioperative data, including 30-day mortality and neurological complications, were retrospectively obtained from our electronic patient's records, including follow-up (FU) data of outpatient clinical visits and computed tomography (CT).

Results: Thirty-day mortality in the entire FET cohort was 16.2\% (AAD 18.7\% vs. non-AAD 11.1\%; $\mathrm{n}=0.414)$. Severe brain injury was the leading cause of death in AAD patients $(12.0 \%$ vs. $0 \%$ non-AAD; $\mathrm{P}=0.030$ ). Overall permanent stroke and spinal cord injury was $12.6 \%$ and $3.6 \%$. Four patients in the AAD group developed paraplegia and permanent stroke rate was significantly higher in AAD compared to non$\mathrm{AAD}$ patients (17.3\% vs. 2.8\%; $\mathrm{P}=0.034)$. One, 3- and 5-year survival rates were $78.7 \% \pm 4.0 \%, 72.2 \% \pm 4.8 \%$, and $64.3 \% \pm 6.8 \%$ for the total cohort; survival at $1-$, 3 - and 5 -year was $76.7 \% \pm 5.0 \%, 71.0 \% \pm 6.1 \%$, and $64.5 \% \pm 8.3 \%$ for the $\mathrm{AAD}$ cohort compared to $83.1 \% \pm 6.3 \%, 75.0 \% \pm 7.9 \%$ and $66.7 \%$ for non-AAD patients $(\mathrm{P}=0.579)$, respectively.

Conclusions: Our single-center experience confirms good early and mid-term survival after the FET procedure in patients presenting with $\mathrm{AAD}, \mathrm{CAD}$ and aneurysm. Future efforts should focus on reduction of severe neurological complication.

Keywords: Aortic arch surgery; frozen elephant trunk procedure; clinical outcomes; acute aortic dissection

Submitted Mar 31, 2020. Accepted for publication Aug 14, 2020.

doi: $10.21037 /$ jtd-20-1531

View this article at: http://dx.doi.org/10.21037/jtd-20-1531

(c) Journal of Thoracic Disease. All rights reserved. 


\section{Introduction}

Despite significant surgical advances the treatment of pathologies involving the aortic arch is challenging and associated with a high mortality and morbidity. Since the first description of the frozen elephant trunk (FET) technique by the Hannover group in 2003 (1), the concept of combining the advantages of the classical elephant trunk (cET) procedure with endovascular stent-graft treatment of the diseased descending aorta in a single procedure using a hybrid stent-graft is increasingly gaining popularity in the surgical community over the last decade (2-9). Another advantage of the FET technique is that it provides, if necessary, a secure landing zone for a subsequent secondstage thoracic endovascular aortic repair (TEVAR) of the remaining descending aorta. Today, several types of stentgrafts, for example non-branched or multi-branched FET prosthesis, are commercially available and indicated for the surgical treatment of complex aortic arch disease, including thoracic aneurysms (TA), acute (AAD) and chronic aortic dissections (CAD) $(10,11)$. Current indications for the FET procedure are constantly reevaluated and the 2019 published consensus paper of European Association of Cardiothoracic Surgery (EACTS) and European Society for Vascular Surgery (ESVS) endorses the FET procedure for various aortic arch diseases based on the evidence from registries and single center reports (12).

This study reviews our 10-year single-center experience with the FET technique in 111 consecutive patients with complex aortic arch disease and analyzed relevant clinical outcomes to evaluate the efficacy and safety of the procedure. We present the following article in accordance with the STROBE reporting checklist (available at http:// dx.doi.org/10.21037/jtd-20-1531).

\section{Methods}

This retrospective study analysed 111 consecutive patients that underwent FET surgery for aortic arch replacement at out centre between 2009 and 2019 using the non-branched E-vita Open Plus (Jotec GmbH, Hechingen, Germany, $\mathrm{n}=18$ ) or the branched Thoraflex ${ }^{\mathrm{TM}}$ Hybrid stent-graft (Vascuthek, Terumo Aortic, Ichinnan, Scotland; n=93). This study conformed to the provisions of the Declaration of Helsinki (as revised in 2013). The protocol was approved by the local institutional review board and individual patient consent was waived due to the retrospective study design. Patients were subdivided into two groups with respect to their underlying disease: acute type A aortic dissection (AAD group: $\mathrm{n}=75$ ) or chronic type A aortic dissection (CAD; $\mathrm{n}=10$ ) and degenerative aneurysm (DA $\mathrm{n}=26$; non-AAD group: $\mathrm{n}=36$ ).

All data were retrospectively extracted from our institutional quality information management system and electronic patient records. Relevant perioperative data were entered into a pseudonymized database. Clinical follow-up (FU) for survival was obtained until January 2020 and based on visits of patients at our outpatient clinic, that is routinely performed after approximately 3 to 6 months following initial surgery and annually thereafter. In cases where outpatient clinic visits were not performed, the cut-off point of the study was set as the date of the last documented visit during $\mathrm{FU}$ after confirming the patient's survival status through contacting the patient himself or his general practioner. If the patient was confirmed alive then he was set as censor. Radiological computed tomography (CT) was available in $87.7 \%$ of survivors (71 of 81 patients) and $75.7 \%$ of the total FET cohort (84 of 111 patients) during $\mathrm{FU}$, respectively.

\section{Indications and surgical technique}

Indication for surgical treatment followed current guidelines for $\mathrm{AAD}, \mathrm{CAD}$ (>14 days from onset of symptoms) or degenerative TA $(12,13)$. All procedures were performed by experienced aortic surgeons that were specifically trained for both FET stent-grafts. Intraoperative management, the choice of a branched or non-branched FET, and surgical technique of FET implantation was left at the discretion of the attending surgeon and has been previously described by our group in detail (14). In brief, right axillary cannulation was preferred in most cases and performed prior to sternotomy. After right atrial cannulation, CPB was initiated and the left heart was always vented. After surgical dissection of the aortic arch and supra-aortic vessels, cold blood cardioplegic cardiac arrest was facilitated. Surgical repair of aortic root pathologies and other concomitant procedures were performed during cooling. Deep $\left(20-24^{\circ} \mathrm{C}\right)$ or moderate $\left(25-30{ }^{\circ} \mathrm{C}\right)$ hypothermic circulatory arrest (HCA) was established depending on the preoperative neurological status or evidence of cerebral malperfusion. Brain protection was achieved by selective bilateral antegrade cerebral perfusion (SACP, $10-15 \mathrm{~mL} / \mathrm{kg} / \mathrm{min}$ ) via the axillary canula and a selective perfusion catheter placed in the left carotid artery and under neuromonitoring using bilateral near-infrared spectroscopy (NIRS). 
The FET stent-graft type (branched or non-branched) and size was chosen after preoperative evaluation of axial CT scans and the degree of supra-aortic vessel involvement as recommended (10). Oversizing was avoided in all $\mathrm{AAD}$ and $\mathrm{CAD}$ patients and final sizing was performed according to the diameter of the true lumen as measured in the preoperative CT scan and after intraoperative sizing. For patients with aneurysm a discrete FET oversizing of approximately $10 \%$ was tolerated in relation to the measured aortic diameter at the level of the landing zone. The available length for the stent-graft was $15 \mathrm{~cm}$ for the E-vita Open and $10 \mathrm{~cm}$ for the Thoraflex Hybrid stentgraft, respectively, to avoid a distal landing zone beyond T8.

The anchoring anastomosis of the FET was usually performed in Zone 2 or 3 with $8-12$ pledge-reinforced mattress polypropylene sutures. After successful FET deployment, lower body perfusion and rewarming was initiated by arterial cannulation of the perfusion graft of the branched FET stent-graft (Thoraflex Anteflow or Plexus stent-graft) or by direct cannulation of the stent-graft for the non-branched E-vita Open prosthesis. Subsequently, the three supra-aortic vessels were anastomosed separately to the three branches of the FET prosthesis (branched technique) or en-bloc (island technique) to the nonbranched FET graft. Finally, the proximal anastomosis was completed, and cardiac perfusion restored. In cases of FET implantation in Zone 2, an extra-anatomic left subclavian artery (LSA) bypass was achieved via an $8 \mathrm{~mm}$ vascular prosthesis anastomosed at the level of the left axillary artery and the ascending aorta, respectively.

\section{Definition of endpoints}

The primary endpoint was all-cause 30-day mortality and cumulative survival at FU. Cardiac or cerebral death was recorded in case of fatal cardiac failure or severe brain injury as clinically assessed by a neurologist and following neuroimaging (CT or MRI). Secondary endpoints included permanent stroke (PND) or spinal cord injury, defined as any new postprocedural stroke or paraparesis/paraplegia persistent at the time of discharge. Transient or permanent recurrent nerve palsy was recorded in the presence of clinical symptoms and after confirmation by an ENT specialist. In addition, any subsequent aortic interventions for relevant pathologies during the study period, including planned second-stage procedures (TEVAR) were recorded. Finally, CT scans were analyzed with respect to aortic diameters, distal FET landing zone below T8, and presence of type Ib endoleaks. Total (>80\%) or partial (20-80\%) false lumen thrombosis was assessed in the last available CT scans after subdividing the descending aorta into three segments (Segment A: arch anastomosis to distal landing zone of the stent-graft; Segment B: distal landing zone to celiac trunk; Segment C: distal to the celiac trunk) as recommended by Shrestha et al. (11).

\section{Statistical analysis:}

Statistical analysis was performed using the SPSS statistical software package (SPSS Version 23, IBM Corp, Armonk, NY, USA). Continuous variables are expressed as mean with standard deviation (SD) or median with interquartile range (IQR). Counts (n) with percentages (\%) are given for categorial variables. Non-parametric data or parametric data was analyzed with the Mann-Whitney U-test or unpaired $t$-test depending on normality. The Chi-square or Fischer's exact test was used to compare categorical variables. Kaplan-Meier survival curves were computed and group comparison performed using the log-rank test. A P value less than 0.05 was considered statistically significant.

\section{Results}

\section{Demographics, preoperative comorbidities and aortic conditions}

Baseline characteristics of the entire FET patient cohort and subgroups are illustrated in Table 1. Median patient age was 64 years, $71.2 \%$ were males, $9.0 \%$ diagnosed with Marfan syndrome and $14.4 \%$ of patients had previous cardiovascular surgery $(8.1 \%$ open surgery; $6.3 \%$ endovascular repair). Approximately $34.2 \%$ of the FET cohort were in critical clinical status at admission, predominantly due to neurological impairment in $36.0 \%$ or unstable hemodynamics requiring catecholamine support in $19.8 \%$ of patients, respectively. Compared to the non-AAD group, patients with $\mathrm{AAD}$ were younger, more frequently males and had undergone less cardiac or aortic surgery ( $33.3 \%$ vs. $5.3 \% ; \mathrm{P}<0.001$ ). More patients with $\mathrm{AAD}$ were in a critical status prior to surgery $(48.0 \%$ vs. $5.6 \%$; $\mathrm{P}<0.001)$ with elevated lactate levels, but logistic EuroSCORE was comparable between groups. Mean diameters of the ascending and all descending aortic segments (Segment A to C) were greater in patients with $\mathrm{CAD}$ and $\mathrm{DA}$ compared the $\mathrm{AAD}$ cohort, without differences with regard to the extent of the aortic disease accompanying aortic valve disease or 
Table 1 Preoperative characteristics of patients $(n=111)$

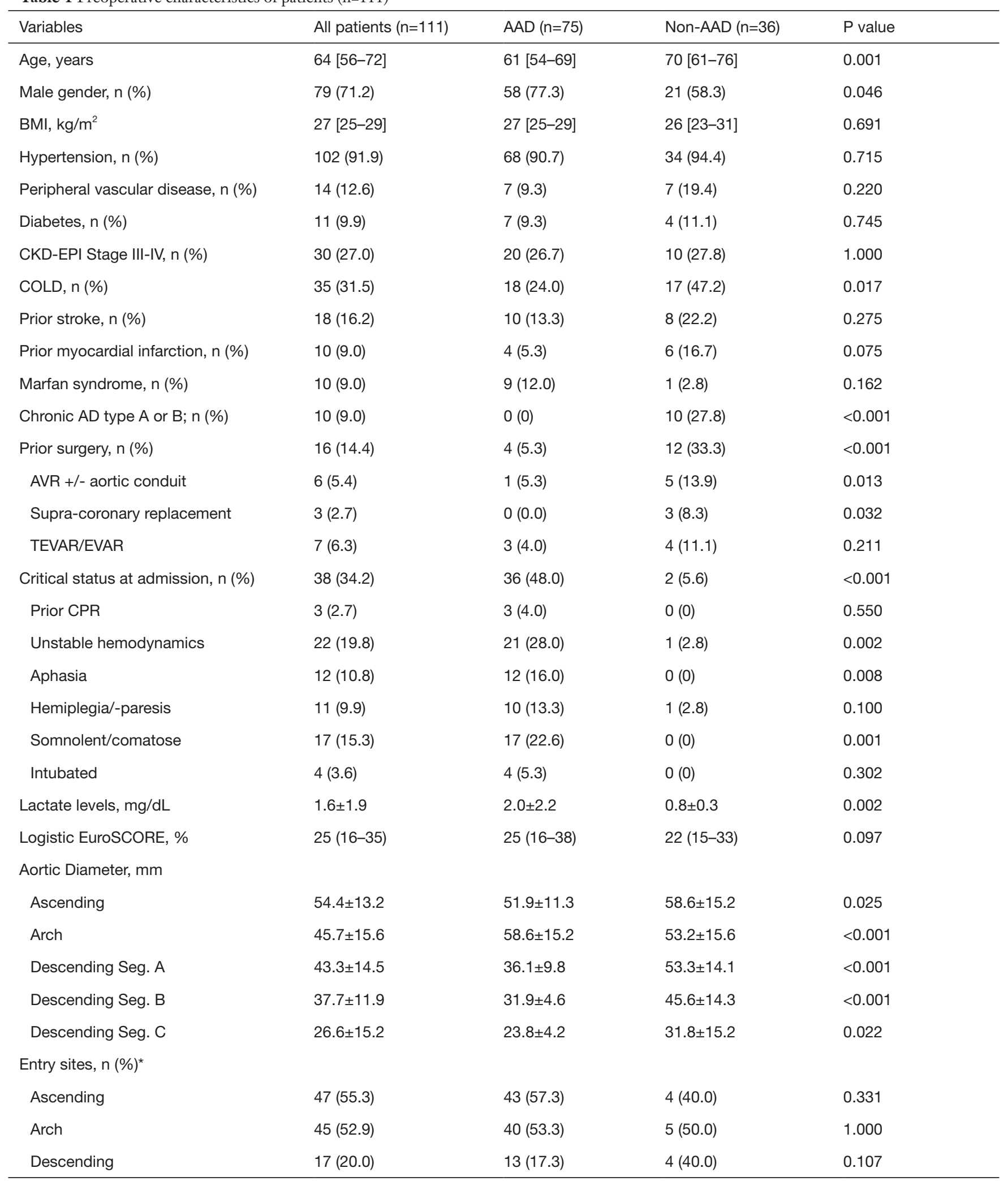

Table 1 (continued) 
Table 1 (continued)

\begin{tabular}{|c|c|c|c|c|}
\hline Variables & All patients $(n=111)$ & AAD $(n=75)$ & Non-AAD (n=36) & $P$ value \\
\hline \multicolumn{5}{|l|}{ Extent of Disease, n (\%) } \\
\hline Arch & $108(97.3)$ & 73 (97.3) & 35 (97.2) & 1.000 \\
\hline Descending & $98(88.3)$ & $64(85.3)$ & $34(94.4)$ & 0.216 \\
\hline Acute aortic rupture, n (\%) & $2(1.8)$ & $0(0)$ & $2(5.5)$ & 0.103 \\
\hline Malperfusion, n (\%) & $37(33.3)$ & $36(48.0)$ & $1(2.8)$ & $<0.001$ \\
\hline Cerebral & $22(19.8)$ & $21(28.0)$ & $1(2.8)$ & 0.002 \\
\hline Mesenteric & $10(9.0)$ & $10(13.3)$ & $0(0)$ & 0.029 \\
\hline \multicolumn{5}{|l|}{ Aortic valve disease, n (\%) } \\
\hline Bicuspid aortic valve & $4(3.6)$ & $4(5.3)$ & $0(0)$ & 0.302 \\
\hline AV stenosis grade $2+$ & $6(5.4)$ & $5(6.7)$ & $1(2.8)$ & 0.662 \\
\hline AV regurgitation grade $2+$ & $50(45.0)$ & $34(45.3)$ & $16(44.4)$ & 1.000 \\
\hline
\end{tabular}

*, only patients with acute (AAD) and chronic aortic dissections (CAD). AAD, acute type A aortic dissection; AD, aortic dissection; AVR, aortic valve replacement; BMI, body mass index; CAD, chronic aortic dissection; CKD-EPI, Chronic Kidney Disease Epidemiology Collaboration; COLD, chronic obstructive lung disease; CPR, cardiopulmonary resuscitation. Data are given as mean (SD), median (interquartile range) or counts (\%).

location of the entry sites in aortic dissection (only AAD or CAD). Clinical signs of cerebral (28.0\%), mesenteric (9.0\%) or limb malperfusion $(25.3 \%)$ was evident in almost half of AAD patients, while only 1 patient with CAD and acute aortic rupture showed unstable hemodynamics and hemiparesis.

\section{Intraoperative data and concomitant procedures}

Intraoperative management and technique are summarized in detail in Table 2. CPB was predominantly instituted via right axillary artery cannulation $(87.1 \%)$, moderate hypothermia $(72.1 \%)$ and bilateral SACP $(100 \%)$, without differences between groups. The Thoraflex Hybrid stentgraft was the preferably used FET prosthesis (83.8\%) and implanted in most cases in Zone 3 (86.5\%), which was comparable between the AAD and non-AAD group, respectively. Proximal and distal stent-graft was significantly greater in the non-AAD compared to AAD group. Supraaortic vessels were anastomosed using the branched technique in $78.4 \%$ of patients, while the Island technique was more frequently used in non-AAD patients compared to AAD patients. Extra-anatomic LSA bypass to the left axillary artery was performed in 8 patients, and the LSA was directly anastomosed to a separate $8-10 \mathrm{~mm}$ vascular graft in the remaining 7 cases.

The need for concomitant procedures was comparable in both groups with $68.5 \%$ receiving supra-coronary replacement of the ascending aorta, $31.5 \%$ aortic valve replacement and $18.0 \%$ additional myocardial revascularization. The need of aortic root repair was more common in $\mathrm{AAD}$ patients ( $30.7 \%$ vs. $11.1 \% ; \mathrm{P}=0.033$ ), who also received more frequently a mechanical valve prosthesis ( $24.0 \%$ vs. $2.8 \% ; \mathrm{P}=0.006$ ) because of their younger age. Consequently, the duration of surgery, $\mathrm{CPB}$ and aortic clamping was significantly reduced in the non-AAD cohort.

\section{Clinical outcomes and FU}

All outcomes measures are presented in Table 3. Overall, 30 -day mortality was $16.2 \%$ and highest in patients with AAD with $18.7 \%$. Mortality rates were $11.5 \%$ in DA (3 of 26 patients) and $10.0 \%$ in CAD patients ( 1 of 10 patients), respectively, resulting to a 30 -day mortality of $11.1 \%$ in 
Table 2 Intraoperative characteristics of patients $(\mathrm{n}=111)$

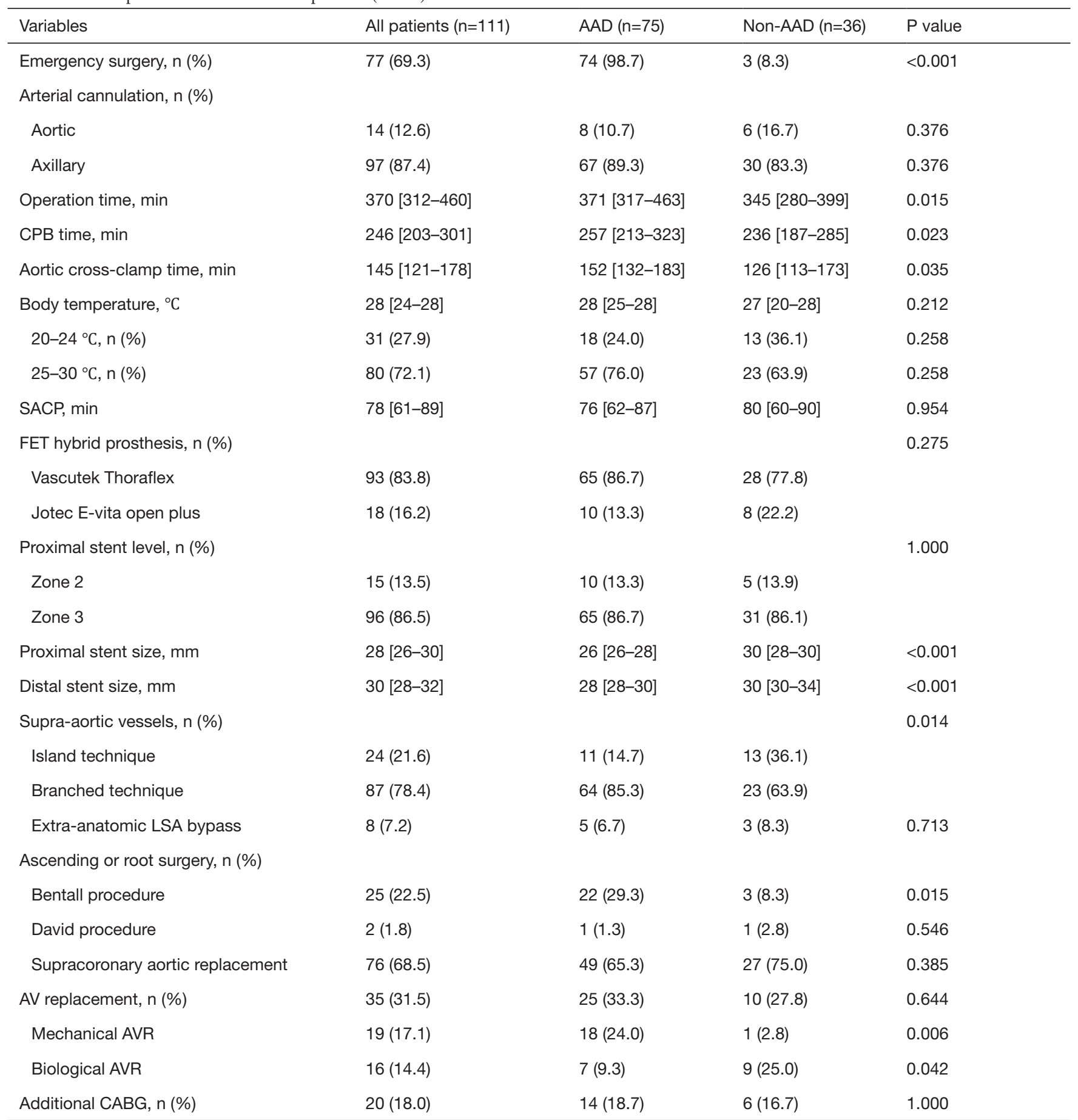

Bentall and David procedure: aortic root procedure with ascending aortic replacement. Data are given as mean (SD), median (interquartile range) or counts (\%). AAD, acute type A aortic dissection; AD, aortic dissection; AKI, AKIN Classification for acute kidney injury; AVR, aortic valve replacement; CABG, coronary artery bypass grafting; CAD, chronic aortic dissection; CPB, cardiopulmonary bypass; RBC, packed red blood cell units; SACP, bilateral selective antegrade cerebral perfusion; supracoronary replacement, supracoronary replacement of the ascending aorta; LSA, left subclavian bypass; FET, frozen elephant trunk. 
Table 3 Postoperative clinical outcome of patients ( $\mathrm{n}=111)$

\begin{tabular}{|c|c|c|c|c|}
\hline Variables & All patients $(n=111)$ & AAD $(n=75)$ & Non-AAD $(n=36)$ & $P$ value \\
\hline Cardiac death & $6(5.4)$ & $5(6.7)$ & $1(2.8)$ & 0.662 \\
\hline Cerebral death & $9(8.1)$ & $9(12.0)$ & $0(0)$ & 0.030 \\
\hline Permanent stroke & $14(12.6)$ & $13(17.3)$ & $1(2.8)$ & 0.034 \\
\hline Recurrent nerve palsy, n (\%) & $22(19.8)$ & $13(17.3)$ & $9(25.0)$ & 0.446 \\
\hline Rethoracotomy for bleeding, n (\%) & $21(18.9)$ & $14(18.7)$ & $7(19.4)$ & 1.000 \\
\hline RBC transfusion $>5$ units & 37 (33.3) & $24(32.0)$ & $13(36.1)$ & 0.673 \\
\hline Laparotomy for GI ischemia, n (\%) & $7(6.3)$ & $6(8.0)$ & $1(2.8)$ & 0.424 \\
\hline Ventilation time, hours & $80[30-301]$ & 81 [31-279] & $70[24-412]$ & 0.083 \\
\hline Ventilation time >96 hrs, n (\%) & $48(43.2)$ & $34(45.3)$ & $14(38.9)$ & 0.546 \\
\hline Tracheostomy, n (\%) & $31(27.9)$ & $20(26.7)$ & $11(30.6)$ & 0.659 \\
\hline ICU stay, days & 9 [5-17] & $10[5-15]$ & 8 [4-21] & 0.073 \\
\hline Hospital stay, days & $18[11-26]$ & $17[10-25]$ & 19 [12-28] & 0.036 \\
\hline Second-stage TEVAR, n (\%) & $21(18.9)$ & $8(10.7)$ & $13(36.1)$ & 0.003 \\
\hline Time to TEVAR, days & 101 [11-229] & 66 [3-320] & $101[21-334]$ & 0.854 \\
\hline Early (in-hospital), n (\%) & $8(7.2)$ & $4(5.3)$ & $4(11.1)$ & 0.434 \\
\hline
\end{tabular}

Data are given as mean (SD), median (interquartile range) or counts (\%). AAD, acute type A aortic dissection; AD, aortic dissection; FET, frozen elephant trunk; GI, gastrointestinal; ICU, intensive care unit; TEVAR, thoracic endovascular aortic repair.

non-AAD patients. The leading cause of death was lifelimiting severe brain injury, which was observed in $12 \%$ of AAD patients ( $\mathrm{P}=0.030$ vs. non-AAD group). Permanent stroke, spinal cord injury and recurrent nerve palsy occurred in $12.6 \%, 3.6 \%$ and $19.8 \%$ of FET patients. Permanent stroke rate was significantly more common in $\mathrm{AAD}$ patients when compared to non-AAD ( $17.3 \%$ vs. $2.8 \% ; \mathrm{P}=0.034)$, while paraplegia due to spinal cord injury occurred in 4 patients, that all had undergone FET implantation for AAD. Length of hospital stay was longer and second-staged TEVAR was more common in the non-AAD, where $36.1 \%$ received additional endovascular treatment of the aorta during index hospitalization (4 patients) or the FU period (9 patients). The main indications for a staged TEVAR approach in AAD patients were as follows: endoleak $\mathrm{Ib}$ $(n=1)$; true lumen collapse $(n=3)$ or progression of the aortic diameter of the downstream aorta $(n=4)$. Following reasons for TEVAR treatment in non-AAD patients were noted: endoleak $\mathrm{Ib}(\mathrm{n}=4)$, late aortic perforation of the distal FET stent $(n=1)$ or planned TEVAR for treatment of thoracoabdominal aneurysm (8).

Postoperative CT scans showed (Table 4) a higher rate of type Ib endoleak in the non-AAD group $(6.5 \% \mathrm{vs}$. $1.9 \% ; \mathrm{P}=0.060$ ). The rate of partial and total false lumen thrombosis in patients with $\mathrm{AAD}$ was higher throughout segment A to C, but only reached statistical difference for segment A compared to patients with CAD. False lumen thrombosis rate decreased significantly in the downstream aortic segments B (60.4\%) and C (56.6\%) when compared to segment $\mathrm{A}(98.1 \%)$ in $\mathrm{AAD}$ patients $(\mathrm{P}<0.001)$.

Mean FU time for the entire FET cohort was $1.8 \pm 1.9$ years (range, 0-9.1 years), representing a total FU of 197.2 
Table 4 Results of postoperative computed tomography (CT) scans ( $\mathrm{n}=84)$

\begin{tabular}{|c|c|c|c|c|}
\hline Variables & All patients & AAD & Non-AAD & $P$ value \\
\hline CT scan after surgery, days & $16[8-197]$ & 14 [7-129] & $35[9-406]$ & 0.634 \\
\hline Distal FET landing zone >T8 & $16.7(14 / 84)$ & $11.3(6 / 53)$ & $25.8(8 / 31)$ & 0.128 \\
\hline Endoleak type Ib, n (\%) & $6.0(5 / 84)$ & $1.9(1 / 53)$ & $6.5(4 / 31)$ & 0.060 \\
\hline \multicolumn{5}{|l|}{ False lumen thrombosis*, n (\%) } \\
\hline Descending Seg. A & 59/63 (93.6) & $52 / 53(98.1)$ & $7 / 10(70.0)$ & 0.011 \\
\hline Descending Seg. B & $37 / 63(58.7)$ & $32 / 53(60.4)$ & $5 / 10(50.0)$ & 0.082 \\
\hline Descending Seg. C & 35/63 (55.6) & $30 / 53(56.6)$ & $5 / 10(50.0)$ & 0.147 \\
\hline
\end{tabular}

*, only patients with acute (AAD) and chronic aortic dissections (CAD).

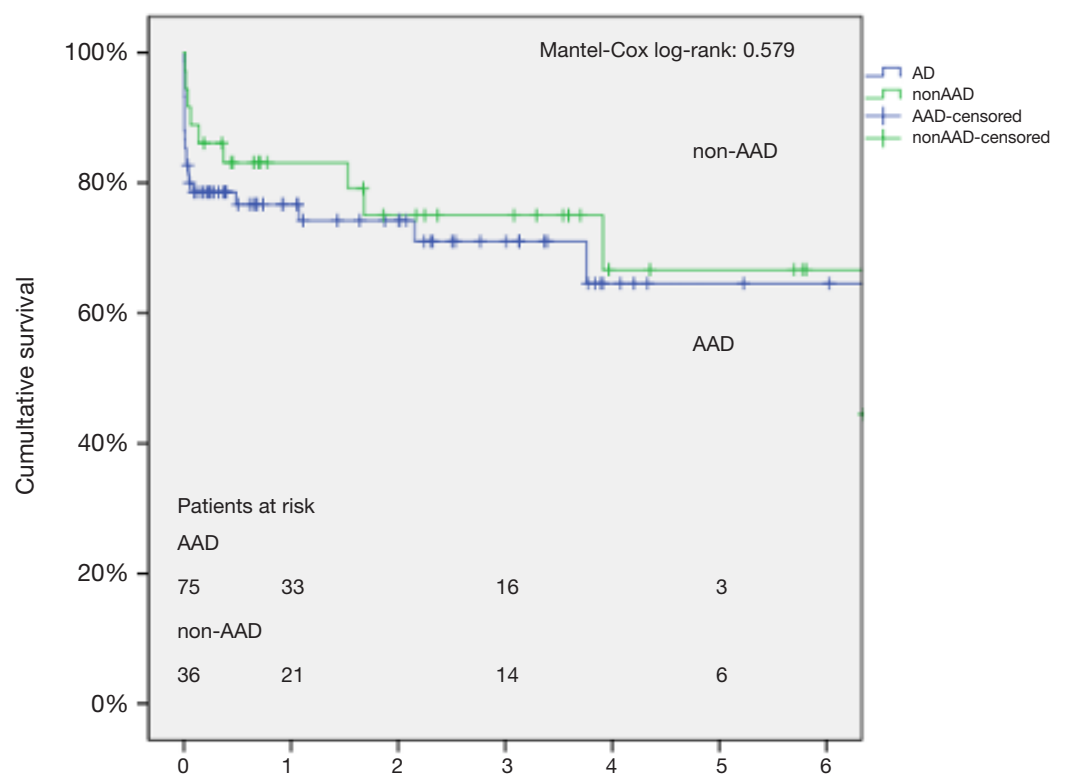

Figure 1 Kaplan-Meier cumulative survival for patients undergoing the FET for patients presenting with (AAD) or without acute (non-AAD) type A aortic dissection.

patient-years (Figure 1). The cumulative 1-, 3- and 5-year survival rates were $78.7 \% \pm 4.0 \%, 72.2 \% \pm 4.8 \%$, and $64.3 \% \pm 6.8 \%$ for the total cohort. Survival at $1-, 3-$ and 5 -year was $76.7 \% \pm 5.0 \%, 71.0 \% \pm 6.1 \%$, and $64.5 \% \pm 8.3 \%$ for the AAD cohort compared to $83.1 \% \pm 6.3 \%$, $75.0 \% \pm 7.9 \%$ and $66.7 \%$ for non-AAD patients (log-rank $\mathrm{P}=0.579)$, respectively.

\section{Discussion}

The present study reviews our institutional experience with the FET procedure for patients with complex aortic arch disease during the last decade. The FET technique was first introduced in our department in 2009 and, since then, two commercially available stent-grafts, the E-vita Open and Thoraxflex Hybrid prostheses, were routinely used for our patients. In this context, the four-branched Thoraxflex Hybrid stent-graft was the most common prosthesis implanted in our series, due its short $(10 \mathrm{~cm})$ stent length and the prespecified arms that enables a more flexible reconstruction in arch pathologies involving the supraaortic vessels (11). In contrast, the non-branched E-vita 
Open or Thoraflex Hybrid stent-grafts was preferred in cases with $\mathrm{DA}$ or CAD were the en-bloc anastomosis of the supra-aortic vessel ("island technique") was feasible.

The overall 30-day mortality of our FET cohort is roughly comparable to other institutional series, that reported in-hospital or 30-day mortality rates ranging from $9 \%$ to $16 \%(4,10,15-17)$. Data from the international multicenter E-vita OPEN-Plus registry showed that the FET procedure was associated to a $15.9 \%$ in-hospital mortality in 509 patients requiring AAD or non-AAD surgery (8). In contrast, a lower $8.8 \%$ operative mortality rate was observed in a recent systematic review of 35 studies with over 3,000 patients undergoing the FET procedure for $\mathrm{AAD}$ and non-AAD surgery (18). The differences among the aforementioned studies with respect to early mortality after the FET procedure can be explained by the differing acuity of the underlying aortic pathology of the included patient cohorts. Indeed, the presence of AAD is known to be an independent predictor for mortality in patients undergoing arch repair (17). Consequently, the reported in-hospital or 30-day mortality of the FET technique in $\mathrm{AAD}$ is consistently higher, ranging from $9 \%$ to $28 \%$, when compared to non-AAD patients requiring mostly elective surgery for CAD or DA (range, $7-13 \%)(8,10,15,18-21)$. Our FET cohort included a high proportion of patients with $\mathrm{AAD}(68 \%)$ that was linked to a $18.7 \%$ early mortality; this influenced the relatively high mortality rate of $16.2 \%$ in our total FET series when compared to previous reports. For example, the proportion of AAD patients in the FET experience from the Hannover or Essen group and the E-vita Open registry was $40 \%, 54 \%$ or $33 \%$, respectively $(8,10,15)$. In addition, the cumulative survival during $\mathrm{FU}$ for our FET cohort was also comparable to previous published mid-term outcome data from larger cohorts showing a 5 -year survival, ranging from $40 \%$ to $75 \%$, for both AAD and non-AAD cohorts $(10,15,17)$.

Despite the potential benefits of the FET technique compared to the classical ET (cET) for aortic arch repair, it is still associated with some significant complications, such as stroke, spinal cord injury and recurrent nerve palsy. Consequently, some experts even discourage the deliberate use of the FET procedure since clinical outcomes are not always superior to the cET, especially for patients with $\mathrm{AAD}$ $(20,22,23)$. The overall prevalence of severe neurological events such as permanent stroke and spinal cord injury ranges between $8.8-15.2 \%$ and $5.6-8.8 \%$ in most singlecenter FET series, respectively, and stroke rates are highest in patients undergoing the FET procedure for AAD (range,
$7-18 \%)(10,15,17)$. These results are also mirrored by data from systematic reviews and large multicentric databases such the E-vita Open and ARCH registry $(8,18,19)$. Consistent to this, the permanent stroke rate in our overall FET cohort was $12.6 \%$, and significantly higher in AAD compared to non-AAD patients (17.3\% vs. $2.8 \%)$. While our stroke rate in AAD patients appears rather high, it should be taken into consideration that $48.0 \%$ of patients of our AAD cohort presented in a critical preoperative status, including neurological impairment, and $28.0 \%$ of patients had evidence of cerebral malperfusion before surgery. In addition, the rate of recurrent nerve palsy was $19.8 \%$ in our series, with $25.0 \%$ for non-AAD patients and $17.3 \%$ for $\mathrm{AAD}$, respectively. These results are quite similar to previous large series and may be related to the predominantly Zone 3 deployment of the FET in most of our patients (86\%) (15).

The occurrence of spinal cord injury leading to paraparesis or paraplegia is a devastating complication and has been directly linked to the FET technique (12). Indeed, various risk factors, including insufficient cooling, prolonged duration of circulatory arrest or inadequate cerebral perfusion, have been identified that explain the higher incidence of spinal cord injury after the FET procedure compared to the $\mathrm{cET}$, while other factors are less well established $(8,24)$. For example, extensive stent coverage of intercostal arteries due to longer stent lengths $(15 \mathrm{~cm})$ or a distal stent-graft landing zone beyond T7-8 was a predictor for SCI in recent studies $(8,19,25)$, but a recent analysis of the ARCH registry failed to confirm this relation (18). Although a strict implementation of bilateral SACP with NIRS monitoring, avoidance of retrograde femoral perfusion, moderate to deep hypothermia and short stent lengths $(83.8 \%$ and $16.7 \%$ of patients with 10 $\mathrm{cm}$ Thoraflex Hybrid graft and distal landing zone $>\mathrm{T} 8$, respectively) were utilized in our FET series, 4 patients with $\mathrm{AAD}$ developed paraplegia due to spinal cord injury in our series. Thus, we cannot rule out that a more rigorous perioperative protocol with regard to routine spinal cord pressure monitoring, lumbar cerebrospinal fluid drainage, lower body perfusion or hypothermia might have benefited these patients (12). Finally, during our clinical FU period approximately $19 \%$ of patients underwent subsequent endovascular treatment of the downstream aorta following FET surgery. Secondary TEVAR at a later stage, was more common in patients with CAD and aneurysm compared to $\operatorname{AAD}(36.1 \%$ vs. $10.7 \%)$, as anticipated, due to the remaining/evolving thoracoabdominal aortic disease and 
higher rate of endoleaks. These observations are closely reflected by the experience of the Hannover and Essen group $(10,15)$. Similarly, our postoperative rate of false lumen thrombosis after FET surgery, for both $\mathrm{AAD}$ and non-AAD patients, underscore the effectiveness of this technique and are in line with previous reports showing a thrombosis rate of up-to $93-99 \%$ around the stent-graft (Segment A) in AAD patients, with progressively decreasing rates in the downstream aortic segments $(4,26,27)$.

The main limitations of our study are its monocentric, retrospective design, the relatively low numbers of patients and incomplete CT scans at FU. Especially the patient numbers for elective arch repair for DA and CAD were low and, therefore, summarized as non-AAD patients to allow a meaningful comparison to $\mathrm{AAD}$ patients. It is certainly acknowledged that $\mathrm{CAD}$ and $\mathrm{DA}$ are different pathological entities and, thus, a separate analysis revealing important differences between these groups would have been desirable. The lack of survival difference in the Kaplan-Meier curve could potentially be attributed to the small study population. In addition, identification of independent predictors or riskadjustment confounding variables using a multivariable logistic regression would have been futile due to the low event rates in our case series. Finally, our observational study cannot rule out a potential patient selection or treatment bias (i.e., surgeon; choice of FET type; surgical technique), that may have influenced our results.

In conclusion, our single-center experience underscores the safety and efficacy of the FET procedure that provides good early and mid-term survival for patients presenting with various aortic arch pathologies including AAD, CAD and aneurysm. Further advancements in the FET technique and perioperative management are necessary to limit neurological complication in this high-risk patient cohort.

\section{Acknowledgments}

Funding: None.

\section{Footnote}

Reporting Checklist: The authors have completed the STROBE reporting checklist. Available at http://dx.doi. org/10.21037/jtd-20-1531

Data Sharing Statement: Available at http://dx.doi. org/10.21037/jtd-20-1531
Conflicts of Interest: All authors have completed the ICMJE uniform disclosure form (available at http://dx.doi. org/10.21037/jtd-20-1531). The authors have no conflicts of interest to declare.

Ethical Statement: The authors are accountable for all aspects of the work in ensuring that questions related to the accuracy or integrity of any part of the work are appropriately investigated and resolved. This study conformed to the provisions of the Declaration of Helsinki (as revised in 2013). The protocol was approved by the local institutional review board and individual patient consent was waived due to the retrospective study design.

Open Access Statement: This is an Open Access article distributed in accordance with the Creative Commons Attribution-NonCommercial-NoDerivs 4.0 International License (CC BY-NC-ND 4.0), which permits the noncommercial replication and distribution of the article with the strict proviso that no changes or edits are made and the original work is properly cited (including links to both the formal publication through the relevant DOI and the license). See: https://creativecommons.org/licenses/by-nc-nd/4.0/.

\section{References}

1. Karck M, Chavan A, Hagl C, et al. The frozen elephant trunk technique: a new treatment for thoracic aortic aneurysms. J Thorac Cardiovasc Surg 2003;125:1550-3.

2. Di Bartolomeo R, Pacini D, Savini C, et al. Complex thoracic aortic disease: single-stage procedure with the frozen elephant trunk technique. J Thorac Cardiovasc Surg 2010;140:S81-5; discussion S6-91.

3. Di Eusanio M, Armaro A, Di Marco L, et al. Short- and midterm results after hybrid treatment of chronic aortic dissection with the frozen elephant trunk technique. Eur J Cardiothorac Surg 2011;40:875-80.

4. Ius F, Fleissner F, Pichlmaier M, et al. Total aortic arch replacement with the frozen elephant trunk technique: 10-year follow-up single-centre experience. Eur J Cardiothorac Surg 2013;44:949-57.

5. Leontyev S, Misfeld M, Daviewala P, et al. Early- and medium-term results after aortic arch replacement with frozen elephant trunk techniques-a single center study. Ann Cardiothorac Surg 2013;2:606-11.

6. Shrestha M, Beckmann E, Krueger H, et al. The elephant trunk is freezing: The Hannover experience. J Thorac Cardiovasc Surg 2015;149:1286-93. 
7. Berger T, Weiss G, Voetsch A, et al. Multicentre experience with two frozen elephant trunk prostheses in the treatment of acute aortic dissectiondagger. Eur J Cardiothorac Surg 2019;56:572-8.

8. Leontyev S, Tsagakis K, Pacini D, et al. Impact of clinical factors and surgical techniques on early outcome of patients treated with frozen elephant trunk technique by using EVITA open stent-graft: results of a multicentre study. Eur J Cardiothorac Surg 2016;49:660-6.

9. Poon SS, Tian DH, Yan T, et al. Frozen elephant trunk does not increase incidence of paraplegia in patients with acute type A aortic dissection. J Thorac Cardiovasc Surg 2020;159:1189-96.e1.

10. Jakob H, Dohle D, Benedik J, et al. Long-term experience with the E-vita Open hybrid graft in complex thoracic aortic diseasedagger. Eur J Cardiothorac Surg 2017;51:329-38.

11. Shrestha M, Kaufeld T, Beckmann E, et al. Total aortic arch replacement with a novel 4-branched frozen elephant trunk prosthesis: Single-center results of the first 100 patients. J Thorac Cardiovasc Surg 2016;152:148-59.e1.

12. Czerny M, Schmidli J, Adler S, et al. Current options and recommendations for the treatment of thoracic aortic pathologies involving the aortic arch: an expert consensus document of the European Association for CardioThoracic surgery (EACTS) and the European Society for Vascular Surgery (ESVS). Eur J Cardiothorac Surg 2019;55:133-62.

13. Erbel R, Aboyans V, Boileau C, et al. 2014 ESC Guidelines on the diagnosis and treatment of aortic diseases:

Document covering acute and chronic aortic diseases of the thoracic and abdominal aorta of the adult. The Task Force for the Diagnosis and Treatment of Aortic Diseases of the European Society of Cardiology (ESC). Eur Heart J 2014;35:2873-926.

14. Sabashnikov A, Heinen S, Deppe AC, et al. Axillar or Aortic Cannulation for Aortic Repair in Patients With Stanford A Dissection? Ann Thorac Surg 2016;102:787-94.

15. Shrestha M, Martens A, Kaufeld T, et al. Single-centre experience with the frozen elephant trunk technique in 251 patients over 15 years. Eur J Cardiothorac Surg 2017;52:858-66.

16. Detter C, Demal TJ, Bax L, et al. Simplified frozen elephant trunk technique for combined open and endovascular treatment of extensive aortic diseases. Eur J Cardiothorac Surg 2019;56:738-45.

17. Leontyev S, Borger MA, Etz CD, et al. Experience with the conventional and frozen elephant trunk techniques: a single-centre study. Eur J Cardiothorac Surg 2013;44:1076-82; discussion 1083.

18. Poon SS, Tian DH, Yan T, et al. Frozen elephant trunk does not increase incidence of paraplegia in patients with acute type A aortic dissection. J Thorac Cardiovasc Surg 2020;159:1189-96.e1.

19. Preventza O, Liao JL, Olive JK, et al. Neurologic complications after the frozen elephant trunk procedure: A meta-analysis of more than 3000 patients. J Thorac Cardiovasc Surg 2020;160:20-33.e4.

20. Shrestha M, Fleissner F, Ius F, et al. Total aortic arch replacement with frozen elephant trunk in acute type A aortic dissections: are we pushing the limits too far?dagger. Eur J Cardiothorac Surg 2015;47:361-6; discussion 6.

21. Verhoye JP, Belhaj Soulami R, Fouquet O, et al. Elective frozen elephant trunk procedure using the E-Vita Open Plus prosthesis in 94 patients: a multicentre French registry. Eur J Cardiothorac Surg 2017;52:733-9.

22. Haverich A. Aortic arch replacement with frozen elephant trunk-when not to use it. Ann Cardiothorac Surg 2013;2:592-6.

23. Bachet J. Open repair techniques in the aortic arch are still superior. Ann Cardiothorac Surg 2018;7:328-44.

24. Leontyev S, Dieterlen MT, Halling M, et al. Evaluation of conventional and frozen elephant trunk techniques on spinal cord blood flow in an animal model. Eur J Cardiothorac Surg 2017;52:485-91.

25. Flores J, Kunihara T, Shiiya N, et al. Extensive deployment of the stented elephant trunk is associated with an increased risk of spinal cord injury. J Thorac Cardiovasc Surg 2006;131:336-42.

26. Berger T, Kreibich M, Morlock J, et al. True-lumen and false-lumen diameter changes in the downstream aorta after frozen elephant trunk implantation. Eur J Cardiothorac Surg 2018;54:375-81.

27. Iafrancesco M, Goebel N, Mascaro J, et al. Aortic diameter remodelling after the frozen elephant trunk technique in aortic dissection: results from an international multicentre registry. Eur J Cardiothorac Surg 2017;52:310-8.

Cite this article as: Liakopoulos OJ, Kroener A, Sabashnikov A, Zeriouh M, Ahmad W, Choi YH, Wahlers T. Singlecenter experience with the frozen elephant trunk procedure in 111 patients with complex aortic disease. J Thorac Dis 2020;12(10):5387-5397. doi: 10.21037/jtd-20-1531 